\section{Structure-function relationship in early diabetic retinopathy: a spatial correlation analysis with OCT and microperimetry}

G Montesano', A Gervasoni ${ }^{1}$, P Ferri ${ }^{1}$, D Allegrini ${ }^{2}$, L Migliavacca ${ }^{1}$, S De Cillà ${ }^{3}$ and L Rossetti ${ }^{1}$

\begin{abstract}
Purpose To study the correlation of the local ganglion cell layer-inner plexiform layer (GCL-IPL) thickness with corresponding retinal sensitivity as studied with microperimetry in patients with Type 2 diabetes and no signs of diabetic retinopathy.

Patients and methods We analyzed 35 healthy subjects (68 eyes) and 26 Type 2 diabetic patients (48 eyes) with no signs of diabetic retinopathy. We tested best corrected visual acuity (BCVA), monocular and binocular constrast sensitivity (CS, Pelli - Robson chart) and retinal sensitivity with microperimetry, and acquired dense macular SD-OCT scans. We then studied the correlation between local GCL-IPL thickness and local sensitivity.
\end{abstract}

Results Mean BCVA was $1.09( \pm 1.03)$ decimals in diabetic subjects and $1.02( \pm 0.15)$ decimals in healthy subjects. Only binocular CS was significantly higher in healthy subjects $(1.18 \pm 0.42$ for healthy subjects, $1.62 \pm 0.63$ for diabetic subjects). In both local and global analysis we observed higher GCL-IPL thickness and higher sensitivity in normal compared with diabetic subjects, but no difference reached significance $(p<0.05)$. Using a mixed multivariate linear model, we found a significant correlation between retinal sensitivity and the correspondent GCL-IPL thickness in diabetic subjects $(0.022 \pm 0.006 \mathrm{~dB} / \mu \mathrm{m}, p=0.0007)$ but not in healthy subjects $(-0.002 \pm 0.006 \mathrm{~dB} / \mu \mathrm{m}$, $p=0.77$ ).

Conclusion despite close similarities between the two groups, we found a significant difference in the structurefunction relationship in diabetic subjects without diabetic retinopathy, suggesting that diabetes might act as an additional effect in the normal deterioration of the visual function related to the inner retina. Eye (2017) 31, 931-939: doi:10.1038/eye.2017.27; published online 3 March 2017

\section{Introduction}

Diabetic retinopathy is the leading cause of blindness in the western world among workingage adults. ${ }^{1}$ Albeit being regarded mainly as a microvascular disorder, ${ }^{2}$ some evidence exist supporting the fact that, in the early phases of retinal involvement, a primary neural damage could exist before the vascular modifications; indeed, early works investigated neural impairment in early diabetic damage to the retinal network ${ }^{3,4}$ focusing on functional analyses, ranging from electroretinographic recordings and perimetry and to color and contrast sensitivity testing. ${ }^{5-7}$

At a cellular level, evidence exists of increased apoptosis in retinas of diabetic subjects. ${ }^{8}$ It also known that, after a primary retinal ganglion cell (RGC) damage, the first modifications start with shrinkage of dendritic arbors, followed by RGC and axon loss. ${ }^{9}$ Recent advancements in the imaging technology, particularly the introduction of Spectral Domain Optical Coherence Tomography (SD-OCT) imaging has allowed a detailed documentation of such anatomical changes in living human subjects, showing a poor resilience of the inner retinal layers to the metabolic stress occurring in diabetic patients, ${ }^{10-13}$ as suggested by the observed thinning of the Ganglion Cell Layer (GCL) and the Inner Plexiform Layer (IPL). This phenomenon could be partially explained by the reduced vascular density and mitochondria concentration in the inner retina; such features, although providing sufficient transparency for
${ }^{1}$ University of Milan, San Paolo Hospital, Milan, Italy

${ }^{2}$ A. Manzoni Hospital, Lecco, Italy

${ }^{3}$ Health Sciences, Università del Piemonte Orientale, Novara, Italy

Correspondence:

G Montesano,

Ophthalmology ASST Santi Paolo e Carlo, University of Milan, Via A. Di Rudinì 8, Milan 20142, Italy Tel: +39 3281644480; Fax: +390250323150. E-mail: giovmontesano@ gmail.com

Received: 10 November 2016 Accepted in revised form: 16 January 2017 Published online: 3 March 2017 
light to reach the photoreceptors, are responsible for the strong dependency of the inner retinal cells on glycolysis rather than oxidative phosphorylation, being de facto less resilient to metabolic and oxidative burdens. ${ }^{14}$ Moreover, early neuronal damage has been implicated in a self sustaining mechanism involving an imbalance of neurotrophic and pro-inflammatory factors, eventually leading to increased vascular leakage and microvascular occlusion. ${ }^{14}$

Despite a wide number of papers investigating early neural alterations in diabetic patients in terms of functional impairment or morphological changes, to our knowledge, only two tried to correlate OCT findings and functional test results. ${ }^{15,16}$ In both these works, diabetic subjects with no or minimal vascular alterations were tested using microperimetry and SD-OCT, but only mean sensitivity and thickness form the foveal location were analyzed. Moreover, correlation between inner retinal layers and retinal sensitivity was not investigated.

In our work we performed, for the first time, a detailed mapping of sensitivities to the corresponding RGC location, in order to calculate a point by point correlation (hereafter denoted as pointwise correlation) of the retinal sensitivity tested with microperimetry and the local thickness of the GCL-IPL complex measured with SDOCT, comparing normal subjects to patients with Type 2 diabetes with no vascular alterations, in an attempt to fill this gap in the knowledge of early alterations in diabetic neuronal damage.

\section{Materials and methods}

This was a cross-sectional study. Type 2 diabetic patients and healthy subjects were selected based on their ophthalmological evaluation performed at our clinic. We enrolled 35 healthy subjects and 26 Type 2 diabetic patients between 40 and 85 year old. This study adhered to the tenets of the declaration of Helsinki, and informed consent was acquired from all participants. This study was approved by the local ethical committee (Comitato Etico Int. Milano Area A).

Required inclusion criteria for Type 2 diabetic patients were the assumption of a medical therapy (oral, insulin, or both) for at least 7 years and no evidence of retinal vascular alterations at fundus examination. Patients under dietetic regimen only were not included in this study. For both groups, exclusion criteria were: refusal to give informed consent, significant media opacities, evidence of other ocular alterations, past history of amblyopia and myopic refractive errors $>5$ diopters. When possible, exclusion criteria were applied to the individual eye and not to the whole patient, but diabetic patients with retinal vascular alterations in at least one eye were excluded.
In addition to the instrumental evaluation described below, we measured Best Corrected Visual Acuity (BCVA, in decimals with a Snellen chart) and contrast sensitivity (with a Pelli - Robson chart). For diabetic patients, we recorded routine blood test results provided by the diabetic patients and performed within 6 months from the inclusion. Raw calculations are usually reported as Mean $\pm S D$, while parameters estimated from the models are reported as Estimate \pm SE.

\section{Microperimetry}

Microperimetry was performed in mesopic conditions with a MAIA 2 Microperimeter (Centervue, Padova, Italy) according to the producer instructions. This device is able to test retinal sensitivity in a range from 0 to $36 \mathrm{~dB}$ by projecting point stimuli. An internal infrared camera allows precise stimuli projection by performing live retinal tracking based on the vessel image and live correction of the stimulus position. We used a 4-2 threshold strategy and, $5^{\circ}$ testing grid, Goldman III stimulus size, 4 asb background luminance. The grid was composed of 3 testing rings of 12 points each, centered on the preferred retinal locus (PRL) of fixation, and with radii of $1^{\circ}, 3^{\circ}$, and $5^{\circ}$, respectively. The reference fundus image used to register stimuli positions was exported, and perimetric data were extracted in a text file and imported in Matlab (The Mathworks, Inc.) using customized analysis codes.

\section{SD-OCT}

Using a Spectralis SD-OCT (Heidelberg Engineering) we performed dense macular cube scans with 145 horizontal scans with $30 \mu \mathrm{m}$ spacing, covering an area of $15 \times 15^{\circ}$ centered on the fovea. All scans were tracked on a Scanning Laser Ophthalmoscopy (SLO) image. HEYEX (Heidelberg Engineering) automated algorithm was used to obtain segmentations of retinal layers. Retinal nerve fiber layer (RNFL), ganglion cell layer (GCL), inner plexiform layer (IPL) and the Bruch membrane segmentation results were visually inspected and, if necessary, manually corrected by an expert blinded to the subject group assignment. However, only gross errors (like inner limiting membrane segmentation passing through the vitreal space or anomalies in the proximity of major vessels) were corrected, trying to keep the automated segmentation as unaltered as possible. Data were then exported in the RAW data format and imported in Matlab (The Mathworks, Inc.) using customized analysis codes. 


\section{Axial length measurement}

Axial length (in $\mathrm{mm}$ ) of each eye was measured via optical interferometry using an IOL Master 500 (Carl Zeiss Meditec, Dublin, CA, Stati Uniti) by averaging five reliable measures.

\section{Data analysis}

Before statistical analysis, we processed raw perimetric data and OCT segmentations in order to obtain coupled measures of sensitivity and local GCL-IPL thickness measures. The first step was to match fundus images from the SD-OCT and microperimetric devices. To this aim, we developed a customized tool in Matlab that allowed an expert user to mark four shared anatomical landmarks on both images and automatically calculated the geometric transformation to remap coordinates of the perimetric fundus image onto the SLO image used to register the SDOCT scans. To compensate for small errors during this remapping procedure, the macular testing grid center was moved to the anatomical fovea (identified as the point with the lowest RGC-IPL thickness within the 6 central degrees of the OCT macular cube), although in no cases the grid was moved by $>1^{\circ}$.

Since the perimetric grid used in this study tested points within $5^{\circ}$ from the fovea, the first complex task was to account for RGC physical displacement from their receptive field. We used the model developed by Drasdo et $a l^{17}$ (as implemented in Turpin et $a l^{18}$ ) to calculate RGC displacement for each one of the tested points. This method compares the cumulative count of ganglion cell receptive fields (calculated using a model estimated from data on normal subjects) and the cumulative count of RGCs (based on the normal density data provided by Curcio and Allen ${ }^{19}$ ) along a specified radial direction, starting from the fovea. It then estimates the RGC displacement from its receptive field as the radial distance between the location of the tested point and the location where the RGC cumulative count reaches the receptive field cumulative count for that tested point. One exemplar result of this calculation, overlaid onto the GCL-IPL thickness map, is shown in Figure 1. Displaced locations were then used to calculate the GCL-IPL thickness location corresponding to each stimulated point.

\section{Statistical analysis}

This was an exploratory study. Since no data on a similar investigation were available a formal sample size calculation could not be performed. Patients were recruited upon arrival to the clinic between September 2015 and September 2016. All data were imported in R (R Foundation for Statistical Computing, Vienna, Austria) for statistical analysis. Linear mixed modeling was used for estimating parameters and assessing significance. Apart from small rotational displacements, the points tested in the different eyes (and their corresponding RGC estimated positions) had almost identical locations with respect to the fovea and were comparable. In this frame, the testing point locations were treated as levels of an unordered factor within the models. This allowed pointwise comparisons and did not force any spatial fitting constraints on the data. Specific models are explained in the Results section. In general, correlations of observations clustered by eye and subject were modeled using nested random effects. Specific predictors of interest and their interactions were tested using likelihood ratio for nested models and only significant components $(p<0.05)$ were kept in the final models. All significances were calculated using this kind of linear models. For subject specific characteristics, with no clustering of observations, this simply reduces to a two sample $t$-test.

\section{Results}

We analyzed 68 eyes from 35 healthy subjects and 48 eyes from 26 subjects with Type 2 diabetes with no retinal vascular alterations. Sample characteristics are summarized in Table 1. Although all efforts have been made to balance the two groups, we found a significant difference in the mean age of the two groups $(58.7 \pm 9.1$ for healthy subjects, $67.19 \pm 9.69$ for diabetic subjects; Mean $\pm S D ; p<0.001)$. For this reason, age was included as a covariate in all the subsequent analyses. Diabetic patients showed, on average, a moderately good glycemic control, with average glycated hemoglobin of $59.75 \pm 18.49(\mathrm{Mean} \pm \mathrm{SD}) \mathrm{mmol} / \mathrm{mol}$, with 11 subjects above $53 \mathrm{mmol} / \mathrm{mol}$ and only 3 above $75 \mathrm{mmol} / \mathrm{mol}$. 10 subjects were under insulin treatment and 16 subjects took oral medications alone. The average duration of treatment was $13.23 \pm 4.67$ years (Mean $\pm \mathrm{SD}$ ).

No differences could be found between healthy and diabetic subjects in terms of BCVA $(p=0.58)$ and axial length $(P=0.98)$.

Since reduced contrast sensitivity has been reported to be one of the first functional impairment, ${ }^{20}$ we used a Pelli - Robson chart to test monocular and binocular contrast sensitivity on healthy and diabetic patients. Monocular tests yielded lower scores (higher contrast sensitivity) for healthy patients, although this difference did not reach significance $(1.91 \pm 0.77$ for healthy subjects, $2.26 \pm 0.78$ for diabetic subjects, Mean \pm SD, $p=0.086$ ), while binocular tests showed a significant difference between the two groups, again the healthy subjects having higher contrast sensitivity $(1.18 \pm 0.42$ for healthy subjects, $1.62 \pm 0.63$ for diabetic subjects, Mean \pm SD, $p=0.002$ ). 
a

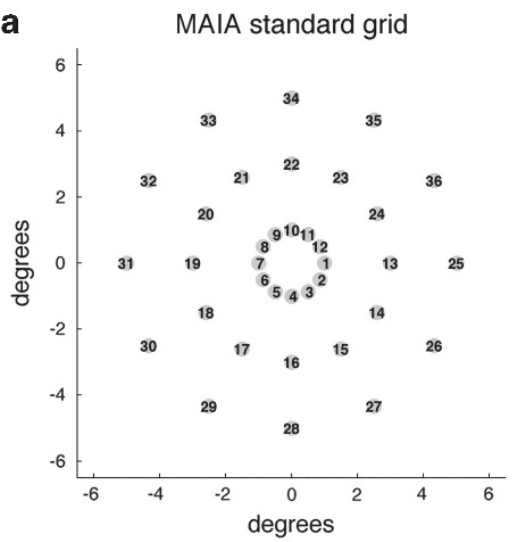

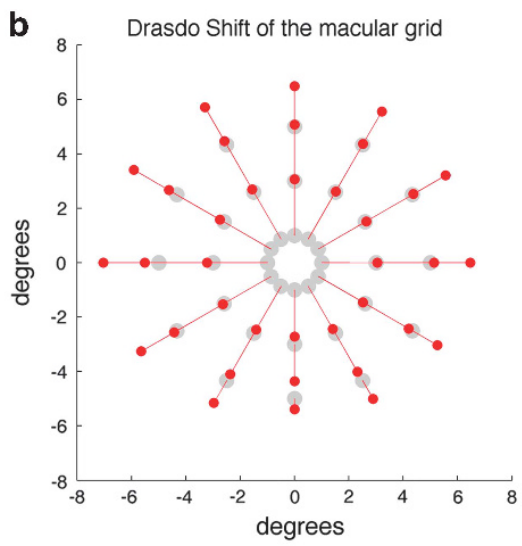

b

C

Shifted sensitivities

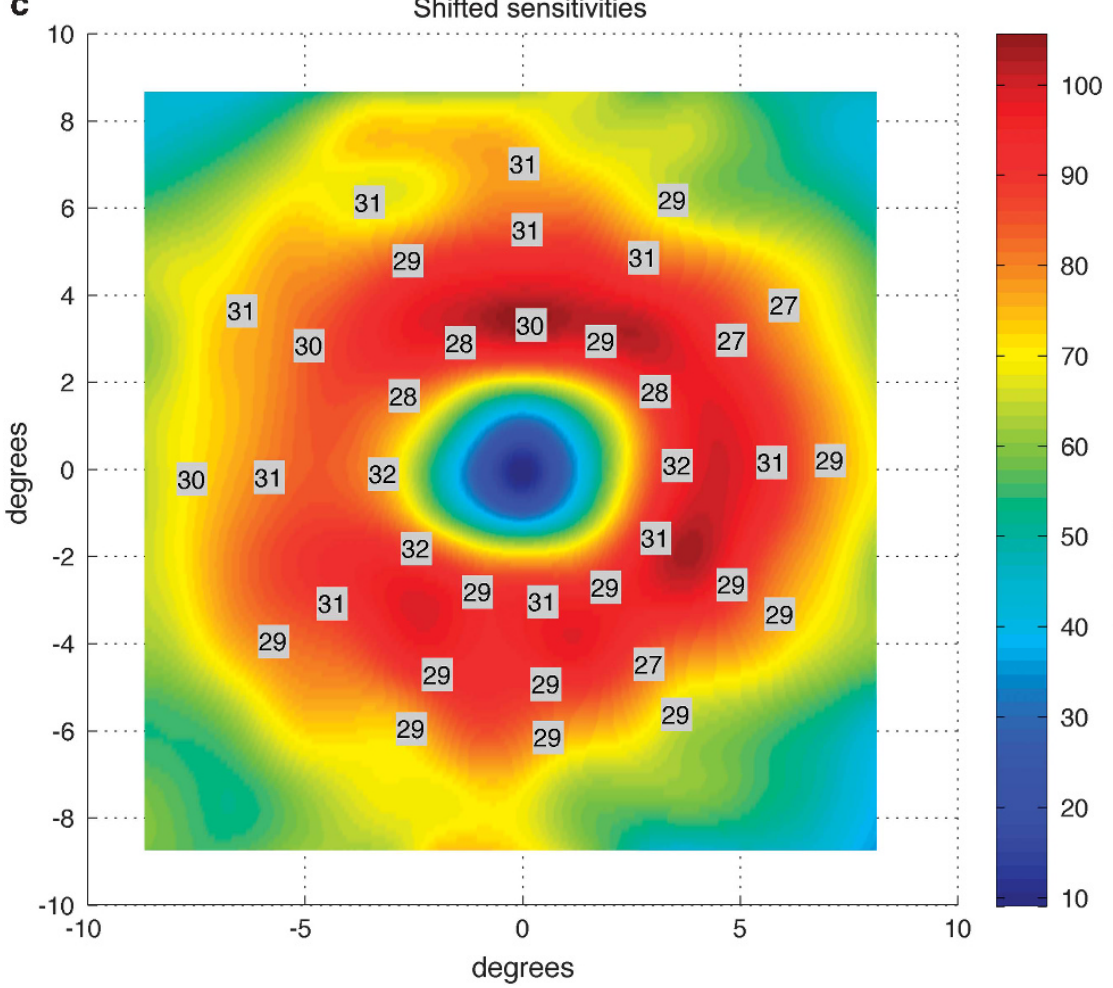

Figure 1 The graphs show the implementation of the Drasdo method for calculating RGC displacement from their respective receptive field. (a) Standard grid of the MAIA 2 perimeter. The gray dots indicate the locations of the tested points, while the overlaid numbers represent the ordinal identifier of the single point. (b) Drasdo shift for all tested points. The gray dots represent the original location of the tested points within the grid, while the red dots represent the corresponding shifted positions according to the Drasdo model, indicating the position of the RGCs responsive to that particular stimulus location. Each original point is joint to the respective shifted point are joined by a red line. (c) Exemplar sensitivities overlaid to the corresponding GCL-IPL thickness map as shifted by Drasdo model.

\section{GCL-IPL Thickness}

We performed pointwise comparisons of the GCL-IPL thickness measured with the SD-OCT between healthy and diabetic subjects. For this analysis, we used the thickness measured at the 36 locations predicted from the Drasdo model for the RGCs receiving the stimuli during the microperimetry test. All calculations were performed using a linear mixed model that included random effects to account for correlations among observations from the same eye and the same subject; fixed effects included the age of the subject (due to the significant age differences between the two groups), the clinical condition (healthy or diabetic), the location on the retina (as an unordered categorical factor), and the interaction between the clinical condition and the retinal location. This formulation of the model allowed an age corrected pointwise comparison of 
the GCL-IPL thickness calculating separate differences for each one of the points analyzed. Results are depicted in Figure 2. Although, the mean thickness was lower in diabetic subjects for most of the points tested (especially for the RGC locations corresponding to the first two-rings of the stimulation grid) only few points (4, flagged with an asterisk on top of the bars, all in the first ring) resulted significant $(p<0.05)$. We also compared overall thickness values at the level of the three different rings (Figure 1) of the testing grid (as shifted by the Drasdo model) using the ring position as a factor in the previous model instead of

Table 1 Descriptive statistics

\begin{tabular}{lccc}
\hline Parameters & $\begin{array}{c}\text { Diabetic } \\
\text { subjects } \\
(N=35)\end{array}$ & $\begin{array}{l}\text { Healthy } \\
\text { subjects } \\
(\mathrm{N}=26)\end{array}$ & P-value \\
\hline $\begin{array}{l}\text { Values per subject: Mean }( \pm S D) \\
\text { Age }\end{array}$ & $67.19( \pm 9.69)$ & $58.7( \pm 9.1)$ & $<0.001$ \\
Glycaemia $(\mathrm{mg} / \mathrm{dL})$ & $147.5( \pm 48.01)$ & & \\
GH (mmol/mol) & $59.75( \pm 18.49)$ & & \\
Therapy & $10(38.5 \%)$ & & \\
$\quad$ Insulin & $16(61.5 \%)$ & & \\
$\quad$ Oral & $13.23( \pm 4.67)$ & & \\
Years of therapy & & & \\
& & & \\
Values per eye: Mean $( \pm S D)$ & $23.4( \pm 0.81)$ & $23.4( \pm 1.3)$ & 0.98 \\
Axial length & $1.09( \pm 1.03)$ & $1.02( \pm 0.15)$ & 0.58 \\
BCVA (decimals) &
\end{tabular}

The table reports mean and SD of meaningful variables for the two groups studied. Fasting glycaemia, glycated hemoglobin (GH), therapy and its duration are reported for diabetic subjects only. Age, axial length, and BCVA were compared between healthy and diabetic subjects and $P$-values are reported on the rightmost column. Only age was significantly different. the point by point location: the greatest differences in the GCL-IPL thickness were on the first ring $(4.42 \pm 2.3 \mu \mathrm{m}$, Estimate $\pm S E, p=0.059)$, although no significant differences below 0.05 could be detected. When this latter analysis was repeated on the GCL thickness alone, only the first parafoveal ring (corresponding to test points at $1^{\circ}$ from the fovea) resulted to be significant with an estimated mean difference of $2.85 \pm 1.4 \mu \mathrm{m}(p=0.045$, Estimate $\pm \mathrm{SE}$, corrected by age). No significant correlation was found with the axial length.

\section{Pointwise sensitivity}

A linear mixed model identical to the one used for the GCL-IPL thickness comparisons in the previous section was used to make pointwise comparisons of the sensitivity data obtained with microperimetry. Again, although the mean sensitivities in diabetic subjects were generally lower, only 4 points, at 3 and $5^{\circ}$ from the fovea, resulted significantly different $(p<0.05)$. Results are depicted in Figure 3. The ring analysis did not yield any significant difference at the level of three rings (all $P$ values above 0.13 , minimum estimated difference $0.6 \mathrm{~dB}$ ).

\section{Pointwise correlation between GCL-IPL thickness and sensitivity}

The main goal of our work was to study the pointwise correlation between the GCL-IPL thickness and the retinal sensitivity. To this aim, we built a linear mixed model. Again, the random part included nested random factors identifying the eye and the subject (to model correlated observations); we use the pointwise sensitivity as

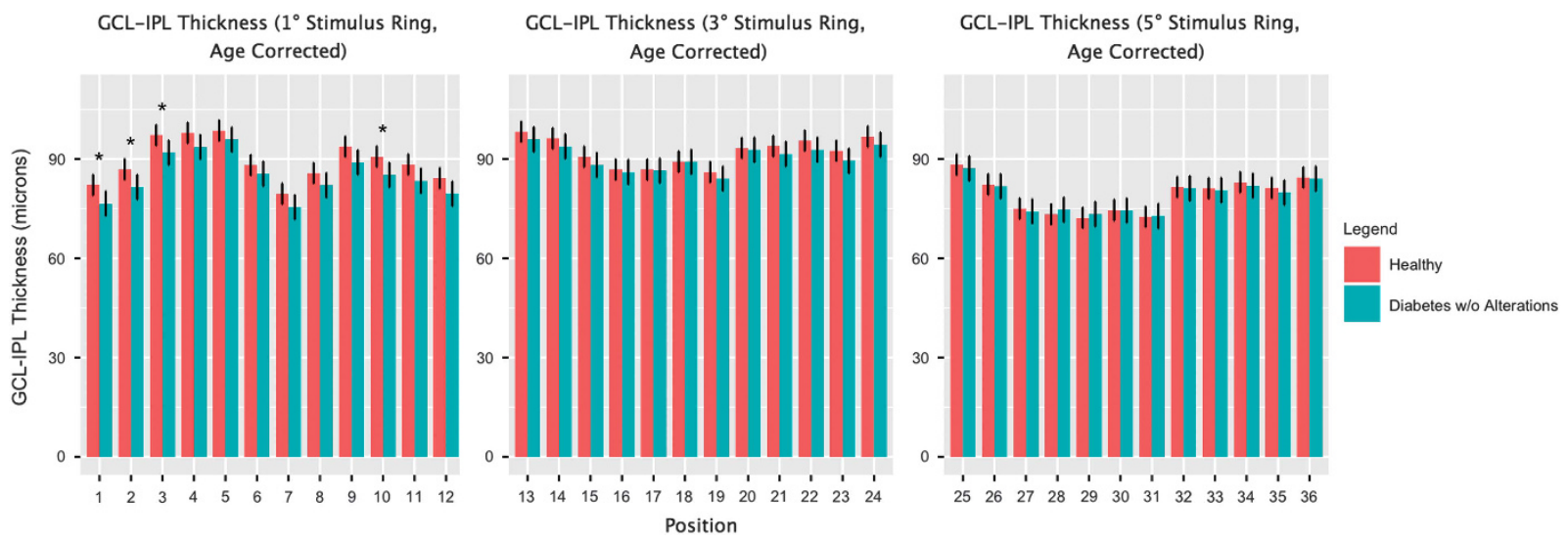

Figure 2 The bar plots show the age corrected estimates of the GCL-IPL thickness at the mean sample age (62). Red bars represent the estimated thickness for a given point in healthy subjects, while green bars represent the estimates for diabetic subjects. Black lines on top of the bars represent the $95 \%$ Confidence Intervals $(95 \% \mathrm{CI})$. Asterisks indicate points where a significant difference $(p<0.05)$ is present. From left to right, the thickness at different radii from the fovea (corresponding to RGC location for stimuli at 1,3 and $5^{\circ}$ ) is presented. Only few points showed significant differences in the inner ring, although most of the points had an estimated lower sensitivity in diabetic subjects. 

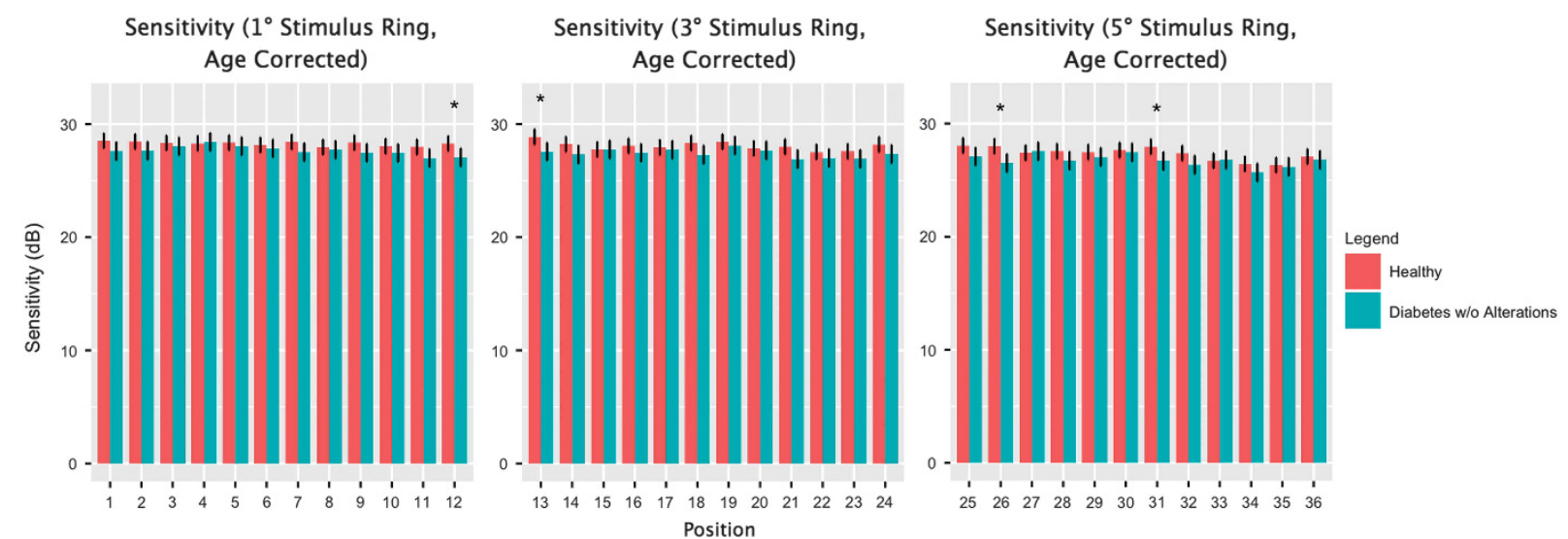

Figure 3 The bar plots show the age corrected estimates of the retinal sensitivities at the mean sample age (62). Red bars represent the estimated sensitivity for a given point in healthy subjects, while green bars represent the estimates for diabetic subjects. Black lines on top of the bars represent the $95 \%$ Confidence Intervals (95\% CI). Asterisks indicate points where a significant difference $(p<0.05)$ is present. From left to right, the sensitivities at different radii from the fovea $\left(1,3\right.$ and $\left.5^{\circ}\right)$ are presented. Only few points showed significant differences, although most of the points had an estimated lower sensitivity in diabetic subjects.

dependent variable and corresponding GCL-IPL thickness, age, clinical condition (healthy or diabetic) and point location (as unordered factor) as predictors. Moreover, all two and three way interactions between the clinical condition, the GCL-IPL thickness and the point location were tested. The three-way interaction and the two-way interaction between GCL-IPL thickness and point location were not significant $(p=0.13$ and 0.20 , respectively) suggesting a homogeneous behavior among all tested points. Then, the final reduced model used for the correlation analysis was the following:

$$
\begin{aligned}
\text { Sensitivity }_{i j k}= & \beta_{0}+\beta_{1} \text { Age }_{k}+\beta_{2} \text { Condition }_{k}+\beta_{3} G C L \\
& - \text { IPLThickness }_{i j k}+\beta_{4} \text { Location }_{i j k} \\
& +\beta_{6} \text { Condition }_{k} \times G C L-\text { IPLThickness }_{i j k} \\
+ & \beta_{7} \text { Condition }_{k} \times \text { Location }_{i j k}+\delta_{k}+v_{j k}+\varepsilon_{i j k} \\
& \varepsilon \sim \mathcal{N}\left(0, \sigma_{1}\right) \\
& \sim \mathcal{N}\left(0, \sigma_{2}\right) \\
& \delta \sim \mathcal{N}\left(0, \sigma_{3}\right)
\end{aligned}
$$

where $\delta_{k}$ and $v_{j k}$ represent the subject and the eye random effects respectively.

This model estimated the age corrected correlation between the points and the local GCL-IPL thickness, calculating two different slopes (for healthy and diabetic subjects respectively) by means of the conditionthickness interaction, homogeneous for all tested points and corrected by age. The sensitivity-thickness slope was $-0.002 \pm 0.006 \mathrm{~dB} / \mu \mathrm{m}$ for healthy subjects and $0.022 \pm 0.006 \mathrm{~dB} / \mu \mathrm{m}$ for diabetic subjects

(Estimate $\pm \mathrm{SE}, p=0.77$ and $p=0.0007$ respectively), being not significantly different from zero for healthy subjects. The estimated difference between these two slopes was significant $(0.024 \pm 0.009 \mathrm{~dB} / \mu \mathrm{m}, p=0.008)$, suggesting a different correlation between sensitivity and GCL-IPL thickness between healthy and diabetic subjects. Results from the model are depicted in Figure 4. The same analysis was performed on GCL thickness alone, yielding similar results: the sensitivity-thickness slope was $0.0006 \pm 0.01 \mathrm{~dB} / \mu \mathrm{m}(p=0.95)$ for healthy subjects and $0.036 \pm 0.01 \mathrm{~dB} / \mu \mathrm{m}(p=0.0006)$ for diabetic subjects. As in the previous case, the difference between the two slopes was significant $(0.035 \pm 0.014 \mathrm{~dB} / \mu \mathrm{m}, p=0.013)$. Since we had correlated predictors within the model, we calculated the variance inflation factor (VIF) on the model excluding the interactions (which are known to create high collinearity even with uncorrelated predictors): the highest VIF value was 2.15, with no evidence of important multicollinearity among predictors.

\section{Discussion}

Our work reports a detailed analysis of anatomical and functional characteristics of diabetic subjects with no retinal alterations. Although a number of papers have addressed these issues in the past, $, 10-12,20,21$ to our knowledge only two works investigated structure-function relationships using OCT and microperimetry. ${ }^{15,16}$ Even so, the relationship between functional alterations and changes in inner retinal layers (which are known to be one the first impaired retinal components in early diabetic damage ${ }^{14}$ ) have not been analyzed. For the first time, we proposed a precise spatial analysis of this relationship by comparing healthy and diabetic subjects with no retinal alterations, with the main aim of studying if the sensitivity of each point tested with microperimetry correlated with the local corresponding 
a

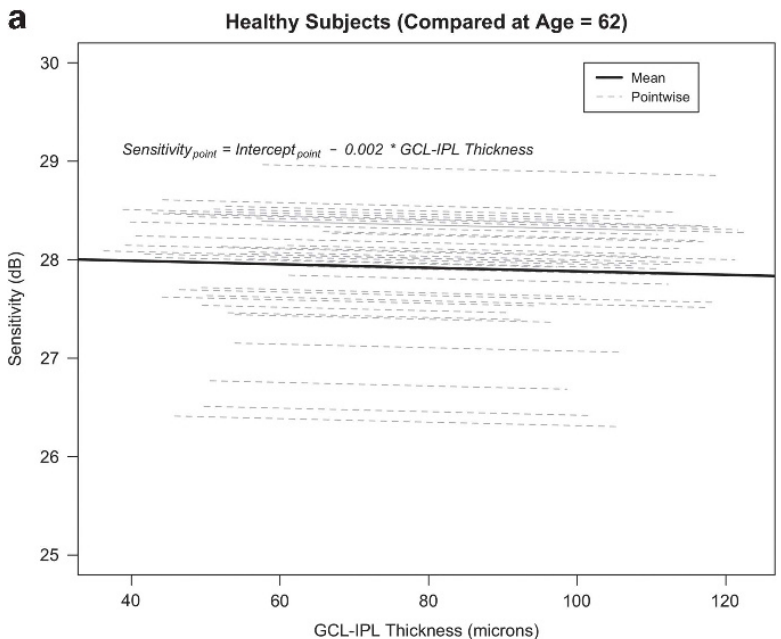

b

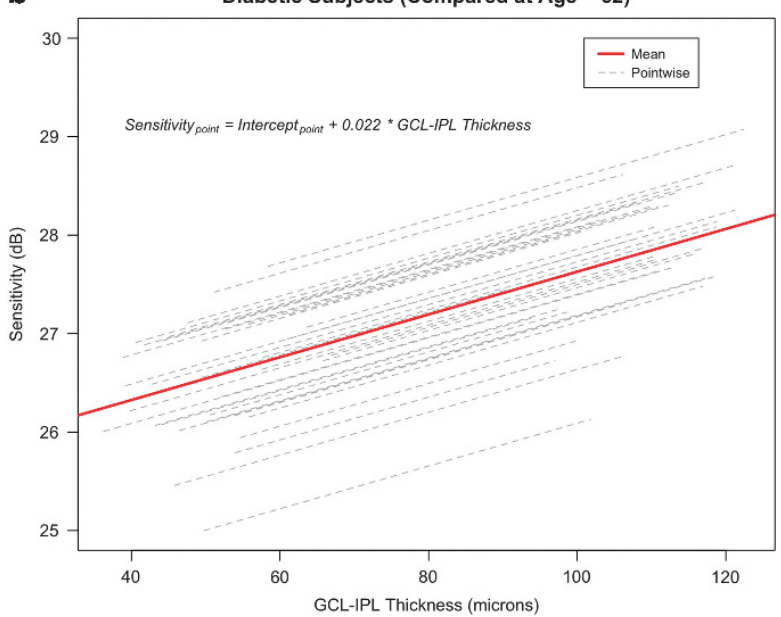

Figure 4 The plots show the relationship between local GCLIPL thickness and pointwise sensitivity. Since all calculations were made using a model corrected by age, the expected values are calculated at the age of 62 , which was the overall sample mean age. (a) The black solid line represents the mean estimated sensitivity by GCL-IPL thickness in healthy subjects; gray dashed lines represent the estimates for each of the tested points. The equation on the top represents the general model for expected sensitivity at a given point in healthy subjects. (b) The red solid line represents the mean estimated sensitivity by GCL-IPL thickness in diabetic subjects; gray dashed lines represent the estimates for each of the tested points. The equation on the top represents the general model for expected sensitivity at a given point in diabetic subjects; the slope was significantly different from that of healthy subjects.

GCL-IPL thickness, in what we called a pointwise analysis.

To test ganglion cell sensitivity we used a microperimeter. Although microperimetry is usually performed to test the functionality of the outer retina, we wanted to take advantage of its fundus tracking technology in order to obtain a precise sensitivity map of a small parafoveal area that would have been heavily affected by the eye movements in a standard perimetry. Indeed, fundus tracking perimetry has been a recently expanding topic in functional testing of the inner retina and has been successfully employed in glaucoma patients even with a larger 24-2 grids. ${ }^{22,23}$

Our mapping strategy relies on the method developed by Drasdo et al ${ }^{17}$ to calculate the RGC displacement from their receptive fields. One limitation of this method is that, as shown by Turpin et al, ${ }^{18}$ it does not account for the inter-subject variability of the RGC foveal displacement, using fixed parameters estimated from normal subjects. Although the same authors provide a method to deal with this issue in normal subjects by modifying the RGC cumulative count based the individual GCL-IPL thickness, their correction cannot be reliably used when investigating subjects with possible pathological modifications of the inner retina, as in our study. In the future, corrections of the Drasdo model based solely on morphometric parameters that are not subjected to pathological variations might provide more accurate calculations.

The two groups compared in this study (healthy and diabetic subjects) showed little differences. There was a significant difference in the mean age of the two groups (8.5 mean year difference). This was partially due to the difficulties in recruiting older healthy subjects. For this reason, age was taken into account in all models used throughout the analysis.

In this frame, we found consistent but very mild differences in terms of GCL and GCL-IPL thickness. The point by point analysis showed that the greatest differences could be located in the points corresponding to the inner stimulation ring, with four points showing statistical significance with p-values below 0.05 . On the other hand, the ring analysis showed a significant difference only in the GCL thickness alone at the inner ring. At this site, the GCL thickness mean difference between healthy and diabetic patients (2.85 (0.10 - 5.6) $\mu \mathrm{m}$, Estimate (95\% CI0) was similar to that reported by Van Dijk et $a l^{11}$ and Carpineto et al ${ }^{12}$ for the perifoveal area $(2.1(-1.6-5.7) \mu \mathrm{m}$, Mean $(95 \% \mathrm{CI})$, from Van Dijk et al) in patients with no diabetic retinopathy, although they reported a non significant difference. The same analysis on the GCL-IPL showed no significant difference (minimum $P$-value $=0.059$ ) but the mean difference at the inner ring $(4.42 \mu \mathrm{m})$ was comparable to the values reported by Carpineto et $a l^{12}(4.6 \mu \mathrm{m})$.

From a functional perspective, we found little differences between the two groups, with no significant differences in terms of BCVA, a slight reduction in retinal sensitivity as tested by microperimetry and a small but significant difference in binocular contrast sensitivity. 
Such mild dissimilarities between the two groups confirm that we were able to detect a class of diabetic patients with very early damage, who might result normal at a general examination.

In spite of this similarity, upon a deeper analysis of the correlation between local inner retinal layer thickness and pointwise sensitivity, we found a significant difference between the two groups: although all tested points showed a homogeneous behavior in terms of structure-function relationship, we found a significant effect of GCL and CGL-IPL thickness on retinal sensitivity in diabetic subjects, while no correlation was evident in the healthy group. This difference suggests that, even when only minimal impairment is detectable, diabetes acts as an additional effect in the normal deterioration of the visual function related to inner retinal layer damage even in the absence of diabetic vascular alterations.

One limitation of this study might lie in the number of subjects recruited, although, as already discussed, our findings are comparable to what has been reported in previous works. It is difficult to compare our findings in terms of retinal sensitivity with the existing literature, since we used a macular grid with a span of $5^{\circ}$ from the fovea with a $36 \mathrm{~dB}$ testing range, which is not comparable mean sensitivities obtained by De Benedetto et $a l^{15}$ (due to the different span of the testing grid) and by Verma et $a^{16}$ (due to the different span and different $\mathrm{dB}$ testing range).

To our knowledge, this is the first attempt to build a spatial analysis correlating inner retinal layer structural and functional modification in early diabetic damage. Spatial models have several advantages over global models, since they are able to assess local characteristics of the process under study, allowing a precise discrimination between uniform and non-uniform modifications. Our quantitative approach could be easily extended to the examination of wider testing grids in order to assess if more peripheral points show a similar homogeneous correlation with inner retinal layer thickness or, on the contrary, to highlight a differential involvement of such points in diabetic neuronal degeneration. Moreover, spatial models could be useful, in a clinical setting, to differentiate between localized inner retinal damages (as in glaucoma) from global retinal degenerations. Indeed, due to the increasing interest in structure-function analysis, accurate models of different physiological and pathological phenomena are required to properly address clinical and research needs.

Our approach might represent step forward in highlighting earlier alterations in diabetic subjects, hopefully offering new insights in understanding, detecting a preventing initial neuronal damage.

\section{Summary}

What was known before

- A wide literature exists supporting the early functional impairment in diabetic subjects prior to the development of the signs of diabetic retinopathy. More recently, since the introduction of SD-OCT, a number of papers reported the early anatomical alterations in the retinas of diabetic patients prior to the development of microvascular alterations, highlighting the involvement of the inner retinal layers in an early neurodegenerative process. Despite this evidence, the function - structure relationship in early diabetic damage has been poorly investigated. Only two papers correlated retinal sensitivity tested with microperimetry to morphological data, but analyzed only global sensitivities and investigated outer retinal layers or central macular thickness.

\section{What this study adds}

- In our work, for the first time, we precisely correlate retinal sensitivity to inner retinal layer thickness in early diabetic patients. We applied a new approach to perform a pointwise correlation analysis overcoming the issues of the spatial displacement of retinal ganglion cells with respect to the stimulus location and allowing a precise mapping of the structure - function relationship.

\section{Conflict of interest}

Luca Rossetti received compensation as a consultant for Centervue, Padua. All other authors have no financial relationships to disclose.

\section{References}

1 Engelgau MM, Geiss LS, Saaddine JB, Boyle JP, Benjamin SM, Gregg EW et al. The evolving diabetes burden in the United States. Ann Intern Med 2004; 140(11): 945-950.

2 Ciulla TA, Amador AG, Zinman B. Diabetic retinopathy and diabetic macular edema: pathophysiology, screening, and novel therapies. Diabetes Care 2003; 26(9): 2653-2664.

3 Wolter JR. Diabetic retinopathy. Am J Ophthalmol 1961; 51: 1123-1141.

4 Bloodworth JM Jr. Diabetic retinopathy. Diabetes 1962; 11: $1-22$.

5 Han Y, Adams AJ, Bearse MA Jr, Schneck ME. Multifocal electroretinogram and short-wavelength automated perimetry measures in diabetic eyes with little or no retinopathy. Arch Ophthalmol 2004; 122(12): 1809-1815.

6 Realini T, Lai MQ, Barber L. Impact of diabetes on glaucoma screening using frequency-doubling perimetry. Ophthalmology 2004; 111(11): 2133-2136.

7 Greenstein VC, Shapiro A, Zaidi Q, Hood DC. Psychophysical evidence for post-receptoral sensitivity loss in diabetics. Invest Ophthalmol Vis Sci 1992; 33(10): 2781-2790.

8 Abu-El-Asrar AM, Dralands L, Missotten L, Al-Jadaan IA, Geboes K. Expression of apoptosis markers in the retinas of human subjects with diabetes. Invest Ophthalmol Vis Sci 2004; 45(8): 2760-2766. 
9 Leung CK, Weinreb RN, Li ZW, Liu S, Lindsey JD, Choi N et al. Long-term in vivo imaging and measurement of dendritic shrinkage of retinal ganglion cells. Invest Ophthalmol Vis Sci 2011; 52(3): 1539-1547.

10 van Dijk HW, Kok PH, Garvin M, Sonka M, Devries JH, Michels RP et al. Selective loss of inner retinal layer thickness in type 1 diabetic patients with minimal diabetic retinopathy. Invest Ophthalmol Vis Sci 2009; 50(7): 3404-3409.

11 van Dijk HW, Verbraak FD, Kok PH, Stehouwer M, Garvin MK, Sonka M et al. Early neurodegeneration in the retina of type 2 diabetic patients. Invest Ophthalmol Vis Sci 2012; 53(6): 2715-2719.

12 Carpineto P, Toto L, Aloia R, Ciciarelli V, Borrelli E, Vitacolonna E et al. Neuroretinal alterations in the early stages of diabetic retinopathy in patients with type 2 diabetes mellitus. Eye 2016; 30(5): 673-679.

13 Chhablani J, Sharma A, Goud A, Peguda HK, Rao HL, Begum VU et al. Neurodegeneration in Type 2 diabetes: evidence from spectral-domain optical coherence tomography. Invest Ophthalmol Vis Sci 2015; 56(11): 6333-6338.

14 Antonetti DA, Lieth E, Barber AJ, Gardner TW. Molecular mechanisms of vascular permeability in diabetic retinopathy. Semin Ophthalmol 1999; 14(4): 240-248.

15 De Benedetto U, Querques G, Lattanzio R, Borrelli E, Triolo G, Maestranzi G et al. Macular dysfunction is common in both type 1 and type 2 diabetic patients without macular edema. Retina 2014; 34(11): 2171-2177.

16 Verma A, Rani PK, Raman R, Pal SS, Laxmi G, Gupta M et al. Is neuronal dysfunction an early sign of diabetic retinopathy? Microperimetry and spectral domain optical coherence tomography (SD-OCT) study in individuals with diabetes, but no diabetic retinopathy. Eye 2009; 23(9): 1824-1830.

17 Drasdo N, Millican CL, Katholi CR, Curcio CA. The length of Henle fibers in the human retina and a model of ganglion receptive field density in the visual field. Vis Res 2007; 47(22): 2901-2911.

18 Turpin A, Chen S, Sepulveda JA, McKendrick AM. Customizing structure-function displacements in the macula for individual differences. Invest Ophthalmol Vis Sci 2015; 56(10): 5984-5989.

19 Curcio CA, Allen KA. Topography of ganglion cells in human retina. J Comp Neurol 1990; 300(1): 5-25.

20 Sokol S, Moskowitz A, Skarf B, Evans R, Molitch M, Senior B. Contrast sensitivity in diabetics with and without background retinopathy. Arch Ophthalmol 1985; 103(1): 51-54.

21 Bearse MA Jr., Han Y, Schneck ME, Barez S, Jacobsen C, Adams AJ. Local multifocal oscillatory potential abnormalities in diabetes and early diabetic retinopathy. Invest Ophthalmol Vis Sci 2004; 45(9): 3259-3265.

22 Rossetti L, Digiuni M, Rosso A, Riva R, Barbaro G, Smolek MK et al. Compass: clinical evaluation of a new instrument for the diagnosis of glaucoma. PLOS ONE 2015; 10(3): e0122157.

23 Fogagnolo P, Modarelli A, Oddone F, Digiuni M, Montesano G, Orzalesi N et al. Comparison of Compass and Humphrey perimeters in detecting glaucomatous defects. Eur J Ophthalmol 2016; 26(6): 598-606. 